\title{
Pediatric diagnostic cerebral angiography: practice recommendations from the SNIS Pediatric Committee
}

\author{
Neeraj Chaudhary, ${ }_{1}^{1}$ Lucas Elijovich 이, ${ }^{2}$ Mesha Martinez, ${ }^{3}$ Johanna T Fifi, ${ }^{4}$ \\ Santiago Ortega-Gutierrez (ㄷ, ${ }^{5}$ Ali Shaibani, ${ }^{6,7}$ Aditya S Pandey (ㄷ) ${ }^{8}$ Shuichi Suzuki, ${ }^{9}$ \\ R Ryan Field, ${ }^{10}$ Joseph J Gemmete (1), ${ }^{11}$ Daniel L Cooke, ${ }^{12}$ Sandra Narayanan, ${ }^{13}$ \\ Steven W Hetts (1) , ${ }^{14}$ Darren B Orbach, ${ }^{15}$ Monica S Pearl (1) ${ }^{16,17}$
}

For numbered affiliations see end of article.

\section{Correspondence to} Dr Monica S Pearl, Radiology, Children's National Hospital, Washington DC 20010, USA; mspearl@childrensnational.org

Received 4 February 2021 Revised 25 March 2021 Accepted 28 March 2021 Published Online First 19 April 2021
Check for updates

(C) Author(s) (or their employer(s)) 2021. No commercial re-use. See rights and permissions. Published by BMJ.

To cite: Chaudhary N, Elijovich L, Martinez M, et al. J Neurolntervent Surg 2021:13:762-766.

\section{INTRODUCTION}

The pediatric population spans a diverse age group, ${ }^{1}$ from neonates and infants to adult-sized teenagers, each with unique physiological characteristics that must be considered when performing diagnostic cerebral angiography (DCA). Herein we refer to pediatric patients as under the age of 18 years. Neonates will not be discussed further due to the very limited role of DCA in the absence of planned neurointervention, most commonly for vein of Galen malformations and other high-flow arteriovenous fistulas leading to life-threatening heart failure.

The number of pediatric neuroangiography procedures performed continues to grow ${ }^{2}$ and it is evident that the technical aspects of performing pediatric DCA are highly variable among practitioners, including our own Task Force members. This reflects the diversity of angiographers from different specialties and the varying levels of prior pediatric neuroangiography training and experience. In 2019, the Society of Neurointerventional Surgery (SNIS) surveyed its membership regarding their individual fellowship training and current practice as it relates collectively to pediatric neuroangiography, including both diagnostic and interventional procedures. Unpublished results indicate that pediatric neuroangiography training and current experience is limited across the nation (figure 1). Most respondents (94\%) performed pediatric DCA during fellowship, though this experience was mostly limited and low volume. In children under 4 years of age, that experience was further reduced (40\%: 0-10 cases; 45\%: 11-49 cases). Despite this limited exposure, $76 \%$ of respondents reported currently treating pediatric patients, though in more than half $(53 \%)$, that volume remains low: 0-10 pediatric cases per year. Practice guidelines for cervicocerebral catheter angiography have been published for adults, ${ }^{3}$ however, no similar recommendations exist for children. Given this information, Pediatric Neurointerventional Task Force Committee members of the SNIS sought to provide a framework with which safe pediatric DCA can be performed, detailing specific procedural considerations as well as peri-procedural evaluation and care.

Despite widespread availability and advances in noninvasive vascular imaging, DCA remains a valuable diagnostic tool and is commonly performed to delineate the angioarchitecture of, and hemodynamic flow patterns for, many cerebrovascular pathologies including stroke, vasculopathy (ie, moyamoya), arteriovenous malformations, arteriovenous fistulas, and aneurysms. DCA has a low complication rate $^{45}$ and maintaining this safety profile in children is an expectation for practitioners performing this procedure. This is predicated on supplementing prior training and experience with a sustained, consistent volume of pediatric cases, while paying special attention to the important nuances herein described.

\section{BEFORE THE PROCEDURE}

\section{Pre-procedure evaluation}

It is critical to review pertinent prior neuroimaging studies and confirm that DCA is indicated. For healthy children without other medical problems, a recent routine history and physical examination, (ie, 30-90 days prior) by their primary care or referring physician suffices for medical evaluation prior to DCA. This information can be updated during the immediate pre-procedure evaluation by the anesthesia and neuroangiography teams on the day of the DCA. Children with cardiopulmonary disease, complex medical histories, or with a history of difficult airway should also be assessed in a pre-operative anesthesia clinic for medical optimization. In select higher-risk patients, such as children with sickle cell anemia and critical arterial stenoses such as moyamoya, additional precautions may need to be implemented, such as preadmission the night prior to the procedure and supplemental hydration while nil per os (NPO). In addition, children with sickle cell anemia may also need a blood transfusion (eg, target hemoglobin of $10 \mathrm{~g} / \mathrm{dL}$ ) to help avoid a sickle cell crisis.

Routine laboratory tests are not universally required for all children undergoing DCA. A complete blood count, basic metabolic panel, and a coagulation profile should be considered in the subset of children with other medical comorbidities and history of coagulopathy, long-term medication intake, or substance abuse. Routine pregnancy testing is required after the onset of menstruation in females.

\section{Contrast allergy risk and prophylaxis}

Allergic reactions to contrast media are independent of contrast dose and occur in less than $3 \%$ 


\section{Numbers of pediatric cases performed}

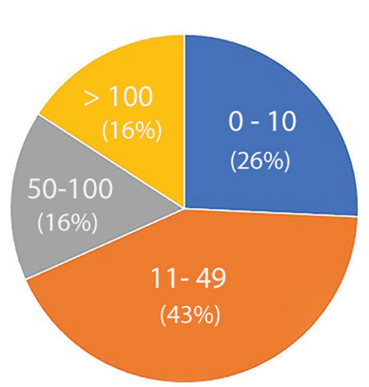

During fellowship

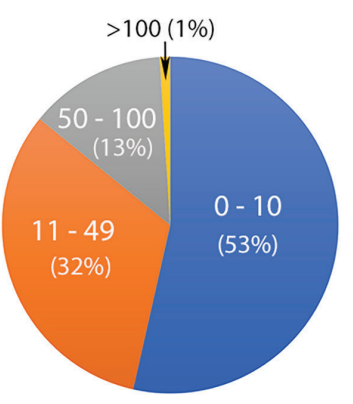

In current practice
Figure 1 Results from a 2019 SNIS membership survey on pediatric experience. 78 members of the SNIS responded to a 13-question survey on fellowship training and current practice involving pediatric patients. Number of cases performed includes both diagnostic and interventional angiographic procedures and sclerotherapy procedures for vascular malformations.

of cases in adults. ${ }^{6}$ In children, the incidence is estimated to be lower with one study of 11306 pediatric intravenous (IV) contrast injections reporting an allergic contrast reaction rate of $0.18 \% .^{7}$ Reactions may range in severity from hives, itching, urticaria rash, and wheezing to more serious reactions including angioedema, bronchospasm, or anaphylaxis, and are reportedly less frequent with low-osmolar non-ionic contrast media, which are the agents of choice. ${ }^{6}$ Several prophylaxis regimens exist to minimize the risk of an allergic reaction, and an example is provided in table 1 .

\section{Contrast agents}

The risk of contrast agent-induced nephropathy in children is low, dose-dependent, and decreases with the use of non-ionic, low-osmolar agents. ${ }^{6}$ Although higher limits up to $8 \mathrm{~mL} / \mathrm{kg}$ have been reported for endovascular interventions in the setting of urgent conditions, ${ }^{6}$ DCA can and should be performed using low volumes of contrast $(2-4 \mathrm{~mL} / \mathrm{kg})$. This can be achieved by tailoring the examination to minimize both the number of vessels catheterized and the number of contrast injections per vessel. Diluting the contrast medium with saline $(1: 1$, that is, half strength) is another effective way to reduce the contrast dose administered while maintaining diagnostic image quality. Adequate oral or IV pre- and post-procedure hydration may also help reduce the nephrotoxicity risk. Sodium bicarbonate infusion and administration of acetylcysteine can be considered to further mitigate the nephrotoxicity risk in select patients, although their use has not been validated in the pediatric population. ${ }^{8}$ Factors that increase the risk of nephrotoxicity include pre-existing renal impairment, dehydration, diabetes mellitus, heart failure, hyperuricemia, and use of nephrotoxic drugs.

\section{Consent process}

A family-centered approach is a core value of pediatrics, and key components of the procedure must be effectively communicated in order to achieve informed consent. The family and the patient, if age-appropriate and mature, should understand the cerebral angiogram in detail including its potential risks, benefits, and alternatives, as well as expected outcomes and the use of general anesthesia. Parents may have different preferences for the degree to which their child participates in this process. Children are often focused on specific aspects of the procedure such as when they can eat, how long they need to lie flat, and when they can resume physical activity.

\section{Fasting and oral medication guidelines}

Pre-procedural fasting guidelines vary among institutions and are constantly evolving ${ }^{9}$ to decrease the duration during which a child is NPO, as prolonged fasting may cause dehydration with subsequent hypotension. ${ }^{1011}$ The patient and caregivers should receive clear instructions regarding essential oral medication use around the procedure, such as antiepileptics, aspirin, or anticoagulants.

\section{PROCEDURAL CONSIDERATIONS}

\section{General anesthesia}

In select circumstances, mature middle adolescents (15-17 years $)^{1}$ may be considered candidates for conscious sedation, if they are cooperative, calm, and can reliably remain motionless during frequent breath holds. Barring that, we recommend the use of general anesthesia (GA) for all pediatric DCAs, with few exceptions, such as a WADA test and balloon test occlusion (if performed with awake neurological testing). GA with the use of a paralytic agent eliminates unexpected body motion, improves image quality, and allows for safe and precise catheter maneuvers, likely shortening the duration of the procedure. ${ }^{211}$ While most of the authors use general endotracheal anesthesia (GETA) for DCA, some have advocated for the use of a laryngeal mask airway (LMA). Choosing between GETA and the use of an LMA is a decision to be made by the individual anesthesia and neuroangiography teams, ensuring that the LMA provides reliable airway protection and minimizes the risk of potential bronchospasm. ${ }^{12-15}$

As a part of a pre-procedure checklist (table 2) and time-out protocol, the neuroangiographer and anesthesiologist should establish open and free communication to discuss key components of the procedure as well as post-procedure care. This includes blood pressure control and fluid management, which are potential critical challenges during the induction and maintenance of GA, and which are particularly important in vulnerable

Table 1 Contrast allergy prophylaxis

\begin{tabular}{|c|c|c|c|c|c|c|c|}
\hline \multicolumn{4}{|l|}{ Elective procedure } & \multicolumn{4}{|c|}{ Emergent procedure } \\
\hline Drug & Dose & Route & Hours before procedure & Drug & Dose & Route & Hours before procedure \\
\hline Prednisone & $\begin{array}{l}0.5-0.7 \mathrm{mg} / \mathrm{kg} \\
(50 \mathrm{mg} \text { max) }\end{array}$ & PO & $\begin{array}{l}13 \\
7 \\
1\end{array}$ & Hydrocortisone & $\begin{array}{l}2 \mathrm{mg} / \mathrm{kg} \\
(200 \mathrm{mg} \text { max })\end{array}$ & IV & $\begin{array}{l}5 \\
1\end{array}$ \\
\hline Diphenhydramine & $\begin{array}{l}1.25 \mathrm{mg} / \mathrm{kg} \\
(50 \mathrm{mg} \text { max) }\end{array}$ & PO & 1 & Diphenhydramine & $\begin{array}{l}1.25 \mathrm{mg} / \mathrm{kg} \\
(50 \mathrm{mg} \text { max) }\end{array}$ & IV, IM, PO & 1 \\
\hline References $^{637}$ & & & & & & & \\
\hline
\end{tabular}




\begin{tabular}{|c|c|c|}
\hline \multicolumn{2}{|l|}{ Airway management } & Radiation protection \\
\hline \multicolumn{2}{|c|}{$\begin{array}{l}\text { GA } \\
\text { Paralytic } \\
\text { Discuss frequent breath-holds }\end{array}$} & $\begin{array}{l}\text { Grids in or out } \\
\text { Select pediatric protocol }\end{array}$ \\
\hline \multicolumn{2}{|c|}{ Non-invasive monitoring devices } & Heparin \\
\hline $\begin{array}{l}\text { Blood pressure cuff } \\
\text { O2 saturation probe } \\
\text { EKG leads } \\
\text { Temperature probe } \\
\text { Twitch monitor }\end{array}$ & $\begin{array}{l}{ }^{*} \text { Ensure devices are } \\
\text { accessible and do } \\
\text { not obscure catheter } \\
\text { visualization }\end{array}$ & $\begin{array}{l}\text { Bolus IV dose after access } \\
\text { Dose for saline flush bags } \\
\text { Protamine availability }\end{array}$ \\
\hline \multicolumn{2}{|l|}{ Invasive monitoring devices } & Recovery plan \\
\hline $\begin{array}{l}\text { Arterial line } \\
\text { Foley }\end{array}$ & $\begin{array}{l}\text { *Not routinely indicated for } \\
\text { elective DCA }\end{array}$ & $\begin{array}{l}\text { Disposition } \\
\text { Immobilization plan: for example, } \\
\text { exmedetomidine }\end{array}$ \\
\hline - Antibiotics & *Not indicated & Any other special concerns \\
\hline
\end{tabular}

patient cohorts, such as those with moyamoya. Physiologic hemodynamic parameters including pulse oximetry, blood pressure, heart rate, and end-tidal capnometry should be monitored in all children under GA. An arterial line and foley catheter are not routinely indicated for elective DCA, however, their use may be considered in critically ill patients. Monitoring and maintaining temperature control is also crucial, especially in the youngest children, who may lose body heat quickly. Use of a supplemental external warming device, a tabletop warmer, and raising room temperature are all methods to maintain warmth. Routine use of antibiotics prior to groin puncture for vascular access is not recommended.

\section{Vascular access}

The common femoral artery is the recommended access site for pediatric DCA. In small children and when the femoral pulse is difficult to palpate, real-time ultrasound is a useful adjunct to visualize the common femoral artery and its bifurcation into the superficial and deep femoral arteries. The SNIS Standards and Guidelines Committee produced a report on transarterial and transvenous access, ${ }^{16}$ in which several recommendations are made including some for pediatric patients. Transradial access has been reported as feasible in children undergoing neuroangiography procedures, though this recent multicenter study also reports high rates of clinically significant radial artery vasospasm (13.1\%) that restricted catheter movements and an $8.2 \%$ conversion rate to femoral access. ${ }^{17}$ Although there is a trend for increasing use of radial access in adults, we do not recommend this access route for pediatric DCA except for rare circumstances, such as in bilateral femoral artery occlusion or unrepaired severe aortic coarctation.

A 4-French pediatric micropuncture set that includes a $4 \mathrm{~cm}$ (or $7 \mathrm{~cm}$ for older children) micropuncture needle is used to perform a single wall puncture. The delicate tissue requires smooth, gentle advancement of the micropuncture wire into the common femoral, external iliac, and common iliac arteries. The slightest resistance to wire advancement should prompt one to assess the micropuncture wire position fluoroscopically and redirect the wire as needed. Multiple repeated attempts to gain access and forceful technique should be avoided. A saved fluoroscopic image showing the access point and expected arterial course of the micropuncture wire is sufficient for documentation (figure 2). Dedicated femoral angiography should be reserved for situations in which there is a concern for an access-related complication or in the less frequent case of a planned closure device. For the latter, a saved fluoroscopic scene after contrast injection or femoral roadmap may be sufficient and reduces

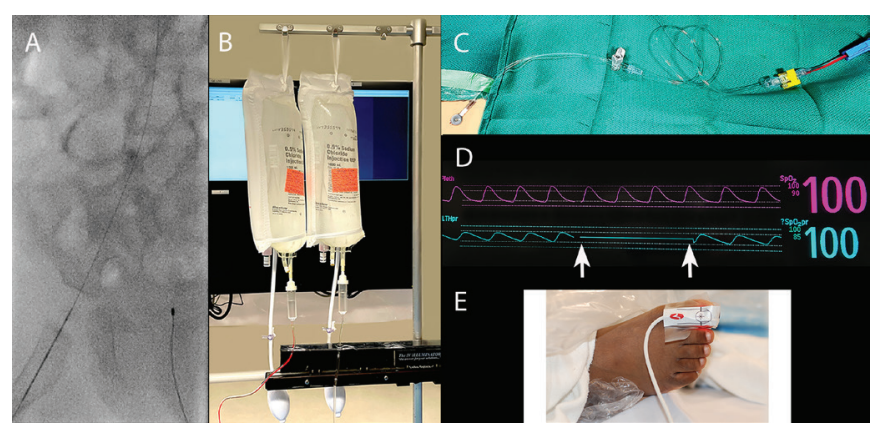

Figure 2 Femoral access-related considerations. A). Saved fluoroscopic image showing the micropuncture needle tip (arrow) over the femoral head and the wire coursing to the patient's left along the expected arterial course. B). Pressurized heparinized saline flush bags are labeled with the heparin concentration. Note the drip chambers are incompletely filled to permit visualization of flow rate through the tubing. Vigilance is required to ensure that the lines are bubble-free. C). The femoral sheath is inserted in the direction of the tubing directed to the flush bags. Care is made to avoid rotating the sheath after insertion. An intervening flow-limiting device is in place between the sheath and heparinized saine. D). Example of normal waveform and it's flattening (between white arrows) during manual compression when excessive pressure is applied. E). A pulse oximeter placed on the great toe of the foot ipsilateral to the femoral sheath allows for monitoring and titration of the level of compression for hemostasis.

radiation exposure. Complications related to vascular access include vasospasm, hematoma, pseudoaneurysm, retroperitoneal hemorrhage, vascular occlusion, and limb ischemia. High rates of femoral artery spasm and subsequent occlusion (9.6\%) had been previously reported in small children undergoing cardiac catheterization $^{18}$, however, amalgamated data from the existing literature on DCA in children found a far lower rate of local groin complications $\left(2.8 \%, 18\right.$ out of 653 patients) ${ }^{19}$ and this should be the expectation for practitioners performing pediatric DCA. Beyond technique itself, a critical feature in the risk of developing vasospasm and subsequent occlusion is the diameter difference between the parent vessel and the sheath or device placed, ${ }^{20}$ which underscores the need to choose the smallest system that can accomplish the goals of the study.

\section{Femoral sheaths}

Routine use of a femoral arterial sheath is recommended as it securely maintains arterial access, minimizes manipulation at the femoral access site, and has a reported lower incidence of bleeding at the puncture site. ${ }^{21}$ Attention should be paid to avoid rotating the femoral sheath after insertion to minimize the risk of vessel injury, particularly in the youngest infants when the difference between the femoral artery diameter and sheath outer diameter (OD) is smallest. Routine DCA and many neurointerventional procedures can be successfully performed using a 4-French system. $^{22}$ The $1.96 \mathrm{~mm}$ OD of a conventional 4-French sheath, however, may still be too large for some of the smallest infants. Newly developed thinner walled 4-French sheaths designed for radial access can be used for femoral access in children. Advantages include the smaller OD $(1.78 \mathrm{~mm})$ without compromising inner diameter, allowing for the use of the existing array of 4-French diagnostic catheters. A recent study on the variation in the OD of common vascular sheaths used in pediatric cardiac catheterization showed that radial sheaths, as a class, have ODs 1-French smaller than the OD of conventional sheaths. Interestingly, the OD of the 4-French Prelude Ideal radial sheath (Merit 
Medical) was found to be smaller than the ODs of both the 3-French (Galt Medical) and 3.3-French (PediaVascular) conventional sheaths, specifically designed for pediatric use. ${ }^{23}$

\section{Heparin}

After femoral access is obtained, the arterial sheath is flushed with heparinized saline and connected to a flow control device limiting the infusion rate through the sheath (figure 2), thereby preventing the inadvertent administration of large volumes of heparinized saline. A systemic heparin bolus of 20-60 units/ $\mathrm{kg}$ is recommended by most Task Force members to reduce the risk of femoral artery thrombosis and catheter-related thrombus formation. This IV bolus dose should also take into account the duration of the procedure and the dose of heparin infused when using a continuous saline flush system, as heparin is also added to the saline flush bags. Heparin doses vary between 1000 and 4000 units of heparin per $1 \mathrm{~L}$ normal saline with lower heparin concentrations used in younger and smaller children: for example, 2000 units of heparin/L NS for those weighing less than $40 \mathrm{~kg}$.

Contraindications to, and precautions for, bolus administration of IV heparin include hypersensitivity, uncontrolled bleeding, recent hemorrhage, thrombocytopenia, severe hepatic, biliary, or renal dysfunction, and recent surgery. Even in these circumstances (except for children with hypersensitivity), the continuous catheter flush solution should be heparinized. Patients with heparin-induced thrombocytopenia should not receive heparin. Argatroban, a synthetic direct thrombin inhibitor, has been used as an alternative anticoagulant in these patients. However, as with many other agents, its safety and effectiveness have not been established in children.

\section{Closed heparinized saline flush systems}

Continuous pressurized flush systems (figure 2) maintain a constant flow through the catheter and may result in less stagnation and less blood loss. Their use, however, requires vigilant monitoring throughout the entire procedure to ensure adequate antegrade flow of bubble-free heparinized saline. Operators must be cognizant of the volume of injected contrast and infused heparinized saline throughout the procedure in order to minimize the risks of nephrotoxicity, volume overload, and hazardously elevated levels of circulating heparin particularly in the smallest children.

Stroke related to iatrogenic embolization is very rare, reported in 14 of $1581(0.9 \%)$ pediatric patients undergoing cerebral angiography in a 1981 study, ${ }^{24}$ with zero or near zero rates reported in several more recent pediatric series. ${ }^{4525}$ As for groin complications, near zero rates of thrombotic complications from DCA should be the expectation for practitioners performing DCA in children.

\section{Radiation dose}

A commitment to radiation protection is a cardinal responsibility for any physician performing cerebral angiography in children, equally as important as the technical aspects of the angiogram itself. Children are more sensitive to stochastic radiation dose effects than adults and implications of this increased vulnerability have been recently described. ${ }^{26}$ Reported radiation doses for DCA range from 350 to $4100 \mathrm{mGy}^{27}$ and this wide variability is largely explained and controlled by operator technique. While intrinsic patient characteristics cannot be altered, default manufacturer settings and operator practices (table 3) can be modified and are highly effective in decreasing radiation dose.
Table 3 Factors and techniques for reducing radiation dose

\begin{tabular}{|c|c|c|}
\hline $\begin{array}{l}\text { Manufacturer settings, tools, } \\
\text { and protocols }\end{array}$ & Basic radiation physics & Operator practices \\
\hline $\begin{array}{l}\text { Biplane } \\
\text { Flat panel detectors } \\
\text { Large display monitor }\end{array}$ & $\begin{array}{l}\text { Remove scatter grids when } \\
\text { possible }\end{array}$ & $\begin{array}{l}\text { Tailor examination: limit number of } \\
\text { vessels catheterized and number of DSA } \\
\text { acquisitions per vessel }\end{array}$ \\
\hline $\begin{array}{l}\text { Familiarize oneself with } \\
\text { manufacturer-specific dose } \\
\text { reduction procedure workflows } \\
\text { Store fluoroscopy, } \\
\text { positions } \\
\text { Overlay } \\
\text { DSA run to roadmap } \\
\text { conversion }\end{array}$ & $\begin{array}{l}\text { Minimize air gap (eg, } \\
\text { raise the table, lower the } \\
\text { detector) }\end{array}$ & $\begin{array}{l}\text { Start with slower ariable frame rates } \\
\text { Prudent use of faster frame rates for high- } \\
\text { flow lesions and as needed }\end{array}$ \\
\hline $\begin{array}{l}\text { Design pediatric protocols: } \\
\text { lower dose per pulse } \\
\text { lower dose per frame }\end{array}$ & Tight collimation & Minimize angulation \\
\hline $\begin{array}{l}\text { Variable frame rate (VFR) } \\
\text { protocols: } \\
\text { lower routine arterial- } \\
\text { phase acquisitions to } 2-3 \\
\text { f/s followed by } 1 \mathrm{f} / \mathrm{s} \text { for } \\
\text { venous phase }\end{array}$ & Filtration (copper) & $\begin{array}{l}\text { Monitor dose during and at the end of } \\
\text { the procedure } \\
\text { Ka,r (mGy) } \\
\text { PKA (in uGym2) } \\
\text { Fluoroscopy time }\end{array}$ \\
\hline References $\left({ }^{2628293839}\right)$ & & \\
\hline
\end{tabular}

Numerous studies highlight specific techniques ${ }^{28}$ that can reduce radiation exposure to children by up to $65 \% .{ }^{29}$

DCA in children should be performed using modern biplane angiography systems equipped with flat-panel detectors. These systems have incorporated advanced technology that includes hardware and software features that enable dose reduction while maintaining diagnostic image quality. Neuroangiographers should be familiar with their equipment configurations and work closely with their radiation physicist, vendor applications specialists, and engineering support to optimize dose settings and create dedicated pediatric protocols. During and after each case, delivered radiation dose parameters (Ka,r in mGy, PKA in $\mu \mathrm{Gym}^{2}$, and fluoroscopy time) should be routinely monitored and recorded. ${ }^{30}$ Establishing one's typical baseline dose parameters for DCA is a valuable practice that allows one to detect and react quickly to anomalously high doses.

\section{Rotational angiography}

Flat-panel CT (FPCT) and 3D or 4D rotational angiography are valuable techniques that may help define anatomic relationships more clearly than conventional $2 \mathrm{D}$ angiography alone and may reduce the need for additional 2D imaging. ${ }^{31}$ In children, the additive value of these applications must be carefully considered as their use entails additional radiation exposure and contrast administration. In terms of contrast, typical rotational angiography injection protocols require more contrast (eg, 18-21 mL for a carotid injection) compared with a standard 2D injection, which may limit the remaining contrast dose available for completion of the DCA. Protocols can be further tailored using lower radiation dose protocols ${ }^{32}$ and various dilutions of contrast (eg, 25\% for FPCT, $50 \%$ for 3D studies) in order to meet the imaging needs and stay within contrast dose limitations.

\section{After the procedure \\ Hemostasis}

Non-occlusive manual compression at the femoral access site for a total of $15-20 \mathrm{~min}$ is the preferred technique for achieving hemostasis with few exceptions for adult-sized teenagers in whom a closure device may be considered. If use of a vascular closure device is planned, one may consider devices that achieve hemostasis without leaving an indwelling intra-vascular component. ${ }^{33}$ In small children, attention must be paid to avoid sustained 
occlusion beyond 1-2 min after removing the femoral sheath in order to minimize the risk of femoral artery occlusion and limb ischemia. A pulse oximeter placed on the ipsilateral great toe can be monitored during compression and used to titrate the degree of compression in order to avoid bleeding at the femoral access site while maintaining a normal arterial waveform (ie, "patent hemostasis") (figure 2).

\section{Bed rest}

Recovery after DCA via a femoral approach involves a variable duration of bed rest with leg immobilization. A conservative approach is a 4-hour period of bedrest after removal of a 4-French sheath when hemostasis is achieved by manual compression. There are, however, reports in adults suggesting that a shorter period of $2-3$ hours is sufficient. ${ }^{34}$ Limiting knee and hip flexion can be aided by tightly tucking a sheet around the leg or securing a padded board to the leg in infants.

Prevention and treatment of emergence delirium or agitation In general, age, type of anesthesia, prior reaction to anesthesia, and pre-procedural behaviors can be used to predict the possibility of emergence agitation or an uncooperative state. ${ }^{35}$ Treatment options include prolonging GA, transitioning to an infusion of sedative medications, or giving a medication bolus at the time of emergence. Recent studies have suggested that $\alpha 2$-adrenergic agonists, e.g., dexmedetomidine, are effective in reducing post-emergence agitation from a volatile anesthetic in both bolus and short infusion forms. ${ }^{35}{ }^{36}$ These $\alpha 2$-adrenergic agonists are effective opioid-sparing alternatives to bolusing narcotics or agents such as midazolam and are not associated with a significant risk of respiratory depression. Adequate pain control is also an important factor to consider in reducing postprocedure agitation, especially in young patients who may have difficulty verbalizing discomfort.

\section{CONCLUSION}

DCA is a safe and increasingly used imaging tool that is practiced by a diverse group of operators with varying levels of prior pediatric neuroangiography training and experience. A consistent volume of pediatric cases as well as modifications in device sizes, medication dosing, radiation protocols, and technique are necessary to maintain the expected favorable safety profile. Referral to a higher-volume pediatric center or practitioner is recommended for those operators who seldom perform cerebral angiography in children.

\section{Author affiliations}

${ }^{1}$ Radiology, University of Michigan Health System, Ann Arbor, Michigan, USA ${ }^{2}$ Departments of Neurology and Neurosurgery, Semmes-Murphey Clinic, Memphis, Tennessee, USA

${ }^{3}$ Neurointerventional Radiology, Indiana University School of Medicine, Indianapolis, Indiana, USA

${ }^{4}$ Neurosurgery, Icahn School of Medicine at Mount Sinai, New York, New York, USA ${ }^{5}$ Division of Neurointerventional Surgery-Interventional Neuroradiology, University of lowa, lowa City, lowa, USA

${ }^{6}$ Radiology, Northwestern University Feinberg School of Medicine, Chicago, Illinois, USA

${ }^{7}$ Radiology, Northwestern Memorial HealthCare Corp, Chicago, Illinois, USA

${ }^{8}$ Neurosurgery, University of Michigan, Ann Arbor, Michigan, USA

${ }^{9}$ Neurosurgery, University of California Irvine, Orange, California, USA

${ }^{10}$ Anesthesia, Neurosurgery, University of California Irvine, Orange, California, USA

${ }^{11}$ Radiology and Neurosurgery, University of Michigan Health System, Ann Arbor, Michigan, USA

${ }^{12}$ Radiology and Biomedical Imaging, University California San Francisco, San Francisco, California, USA

${ }^{13}$ Neurology, University of Pittsburgh, Pittsburgh, Pennsylvania, USA

${ }^{14}$ Radiology, UCSF, San Francisco, California, USA
${ }^{15}$ Neurointerventional Radiology, Boston Children's Hospital, Boston, Massachusetts, USA

${ }^{16}$ Radiology, Children's National Hospital, Washington, District of Columbia, USA

${ }^{17}$ Radiology and Pediatrics, The George Washington University School of Medicine and Health Sciences, Washington, District of Columbia, USA

\section{Twitter Sandra Narayanan @SandraNarayanan}

Contributors This work was a collective effort with all authors contributing to the design, content, and drafting of the manuscript. NC, DO, and MSP revised the manuscript for important intellectual content. All authors approved of the final version and agree to be accountable for all aspects of the work.

Funding The authors have not declared a specific grant for this research from any funding agency in the public, commercial, or not-for-profit sectors.

Competing interests None declared.

Patient consent for publication Not required.

Provenance and peer review Not commissioned; externally peer reviewed.

\section{ORCID iDs}

Lucas Elijovich http://orcid.org/0000-0003-1979-2848

Santiago Ortega-Gutierrez http://orcid.org/0000-0003-3744-7845

Aditya S Pandey http://orcid.org/0000-0003-0789-4273

Joseph J Gemmete http://orcid.org/0000-0003-2760-3613

Steven W Hetts http://orcid.org/0000-0001-5885-7259

Monica S Pearl http://orcid.org/0000-0001-9514-8902

\section{REFERENCES}

1 Hardin AP, Hackell JM, Committee on Practice and Ambulatory Medicine. Age limit of pediatrics. Pediatrics 2017; 140:e20172151.

2 Ashour R, Orbach DB. Interventional neuroradiology in children: diagnostics and therapeutics. Curr Opin Pediatr 2015;27:700-5

3 Radiology ACo. ACR-ASNR-SIR-SNIS practice parameter for the performance of diagnostic cervicocerebral catheter angiography in adults 2016.

4 Burger IM, Murphy KJ, Jordan LC, et al. Safety of cerebral digital subtraction angiography in children: complication rate analysis in 241 consecutive diagnostic angiograms. Stroke 2006;37:2535-9.

5 Lin N, Smith ER, Scott RM, et al. Safety of neuroangiography and embolization in children: complication analysis of 697 consecutive procedures in 394 patients. J Neurosurg Pediatr 2015;16:432-8.

6 Heran MKS, Marshalleck F, Temple M, et al. Joint quality improvement guidelines for pediatric arterial access and arteriography: from the societies of interventional radiology and pediatric radiology. Pediatr Radiol 2010:40:237-50.

7 Dillman JR, Strouse PJ, Ellis JH, et al. Incidence and severity of acute allergic-like reactions to i.v. nonionic iodinated contrast material in children. AJR Am J Roentgenol 2007; 188:1643-7

8 Weisbord SD, Gallagher M, Jneid $H$, et al. Outcomes after angiography with sodium bicarbonate and acetylcysteine. N Engl J Med 2018;378:603-14.

9 Rosen D, Gamble J, Matava C, et al. Canadian Pediatric Anesthesia Society statement on clear fluid fasting for elective pediatric anesthesia. Can J Anaesth 2019;66:991-2.

10 Landrigan-Ossar M, McClain CD. Anesthesia for interventional radiology. Paediatr Anaesth 2014;24:698-702.

11 Lederer W, Grams A, Helbok R, et al. Standards of anesthesiology practice during neuroradiological interventions. Open Med 2016;11:270-8.

12 Keller C, Brimacombe J, Bittersohl J, et al. Aspiration and the laryngeal mask airway: three cases and a review of the literature. Br J Anaesth 2004;93:579-82.

13 Tsai T-Y, Chang P-J, Chen S-H, et al. Severe bronchospasm during laryngeal mask airway placement in an infant. Acta Anaesthesiol Taiwan 2006;44:39-42.

14 Castioni CA, Amadori A, Bilotta F, et al. Italian consensus in neuroradiological anesthesia (ICONA). Minerva Anestesio/ 2017;83:956-71.

15 Levati A, Paccagnella F, Pietrini $D$, et al. SIAARTI-SARNePI guidelines for sedation in pediatric neuroradiology. Minerva Anestesiol 2004;70:698-715.

16 Starke RM, Snelling B, Al-Mufti F, et al. Transarterial and transvenous access for neurointerventional surgery: report of the SNIS standards and guidelines Committee. J Neurointerv Surg 2020;12:733-41.

17 Srinivasan VM, Hadley CC, Prablek M, et al. Feasibility and safety of transradial access for pediatric neurointerventions. J Neurointerv Surg 2020;12:893-6.

18 Alexander J, Yohannan T, Abutineh I, et al. Ultrasound-guided femoral arterial access in pediatric cardiac catheterizations: a prospective evaluation of the prevalence, risk factors, and mechanism for acute loss of arterial pulse. Catheter Cardiovasc Interv 2016:88:1098-107.

19 McCrea N, Robertson F, Ganesan V. Towards evidence based medicine for paediatricians. question 2: neurological complications of diagnostic cerebral catheter angiography in children. Arch Dis Child 2014;99:483-5.

20 Franken EA, Girod D, Sequeira FW, et al. Femoral artery spasm in children: catheter size is the principal cause. AJR Am J Roentgenol 1982;138:295-8. 
21 Moran CJ, Milburn JM, Cross DT, et al. Randomized controlled trial of sheaths in diagnostic neuroangiography. Radiology 2001;218:183-7.

22 Gross BA, Orbach DB. Addressing challenges in $4 \mathrm{~F}$ and $5 \mathrm{~F}$ arterial access for neurointerventional procedures in infants and young children. J Neurointerv Surg 2014;6:308-13.

23 Mathis C, Romans R, Divekar A. Variation in the outer diameter of vascular sheaths commonly used in infant cardiac catheterization. Catheter Cardiovasc Interv 2020:96:620-5.

24 Pettersson H, Fitz CR, Harwood-Nash DC, et al. latrogenic embolization: complication of pediatric cerebral angiography. AJNR Am J Neuroradiol 1981;2:357-61.

25 Hoffman CE, Santillan A, Rotman L, et al. Complications of cerebral angiography in children younger than 3 years of age. J Neurosurg Pediatr 2014;13:414-9.

26 Orbach DB, Stamoulis C, Strauss KJ, et al. Neurointerventions in children: radiation exposure and its import. AJNR Am J Neuroradio/ 2014;35:650-6.

27 Struelens L, Vanhavere F, Bosmans H, et al. Skin dose measurements on patients for diagnostic and interventional neuroradiology: a multicentre study. Radiat Prot Dosimetry 2005;114:143-6.

28 Pearl MS, Torok C, Wang J, et al. Practical techniques for reducing radiation exposure during cerebral angiography procedures. J Neurointerv Surg 2015;7:141-5.

29 Schneider T, Wyse E, Pearl MS. Analysis of radiation doses incurred during diagnostic cerebral angiography after the implementation of dose reduction strategies. J Neurointerv Surg 2017;9:384-8.

30 Raelson CA, Kanal KM, Vavilala MS, et al. Radiation dose and excess risk of cancer in children undergoing neuroangiography. AJR Am J Roentgenol 2009;193:1621-8.
31 Struffert $\mathrm{T}$, Hauer M, Banckwitz R, et al. Effective dose to patient measurements in flat-detector and multislice computed tomography: a comparison of applications in neuroradiology. Eur Radiol 2014;24:1257-65.

32 Pearl MS, Torok C, Katz Z, et al. Diagnostic quality and accuracy of low dose 3D-DSA protocols in the evaluation of intracranial aneurysms. J Neurointerv Surg 2015:7:386-90.

33 Shokuhfar T, Hurley MC, Al-Smadi A, et al. MynxGrip vascular closure device use in pediatric neurointerventional procedures. J Neurosurg Pediatr 2018;21:46670.

34 Tonetti DA, Ferari C, Perez J, et al. Validation of an extrinsic compression and early ambulation protocol after diagnostic Transfemoral cerebral angiography: a 5-year prospective series. J Neurointerv Surg 2019;11:837-40.

35 Mason KP. Paediatric emergence delirium: a comprehensive review and interpretation of the literature. Br J Anaesth 2017;118:335-43.

36 Sun L, Guo R, Sun L. Dexmedetomidine for preventing sevoflurane-related emergence agitation in children: a meta-analysis of randomized controlled trials. Acta Anaesthesiol Scand 2014:58:642-50.

37 Greenberger PA, Patterson R. The prevention of immediate generalized reactions to radiocontrast media in high-risk patients. J Allergy Clin Immunol 1991:87:867-72.

38 Morris PP, Geer CP, Singh J, et al. Radiation dose reduction during neuroendovascular procedures. J Neurointerv Surg 2018;10:481-6.

39 Gailloud P. A large display is a powerful tool to reduce radiation exposure during single-plane fluoroscopically guided procedures. AJR Am J Roentgenol 2015;204:W483-5. 